\title{
Research at the University of Sydney.
}

THE various centres engaged in productive scientific work are continually confronted with the problem of how best to bring the results to the notice of their colleagues. In chemical circles there seems to be a great effort made to confine publication, in the main, to certain well-known journals, to which all chemists will then naturally turn. So long as other channels exist for the chemical "cubist" to express himself in the possibility that he may be the conservative leader of the chemical theorists of the next generation, this is possibly a practical solution, at any rate for the countries where science is now well established.

Overseas universities and research centres frequently adopt the plan of issuing the work of their members, either as reprints from journals, or in local publications, and circulating this to other centres throughout the world with the view of receiving other publications in exchange. Such exchanges are probably growing in favour as a method of building up a library. The University of Sydney began thus to issue reprints in I 894, and in I922 it much enlarged the scope of the scheme, deciding to issue these reprints in various sections dealing with different subjects. A number of these volumes has recently reached us, and though we cannot attempt a complete survey of their contents, we are glad to direct attention to a few of the valuable papers in them.

Series 6 is devoted to geological and geographical papers and vol. I includes twenty memoirs, by members of the staff and scholars of the University, mostly on the geology of New South Wales. The paper of most general interest is the lecture by Sir Edgeworth David on the antiquity of man in Australia, with special reference to the Tasmanians. $\mathrm{He}$ adopts the view that the Australians and Tasmanians come from different stocks, and in reference to the Talgai skull quotes Sir Arthur Keith's view that it is Tasmanian in affinity, though that view is not expressed in Sir Arthur Keith's last discussion of this skull. Prof. Griffith Taylor's presidential address to the Geographical and Historical section of the Australasian Association discusses the bearings of Australian geography on national problems, and adopts the conclusion that owing to the ethnologic superiority of the Chinese to the European, their entry and intermarriage with the Australians would improve the stock. Prof. Benson discusses the correlation of the Australian Carboniferous with the European, and remarks that the Burindi Series has some Viséan affinities. Prof. L. A. Cotton describes the Kurrajong earthquake of August I9I9. Mr. W. R. Browne, in addition to a paper on igneous rocks in South Australia, gives a valuable account of the petrology of the country around the Broken Hill mining field. G. D. Osborne and W. R. Browne describe a beautiful glacially striated pavement of Carboniferous age in New South Wales.

The first volume of Series 9 (Medical Sciences-Non-clinical) of the "Sydney University Reprints" consists of a number of papers most of which have appeared in various publications such as the Journal of Anatomy, the Australian Medical Journal, and the like. One, dealing with the diet of the Australians, by Dr. H. S. Halcro Wardlaw, is an official publication of the New South Wales Board of Trade. Dr. Wardlaw has also reprinted here a valuable paper on the energy consumption of Australian students. Among these contributions to science by members of the University of Sydney, which range in date from I92I to I924, we welcome several by the late Dr. J. I. Hunter, whose premature death has been a great loss to science; these include a study of a Neanderthoid Australian skull, written in conjunction with Mr. A. St. N. Burkitt, and a further contribution to the controversy on the relation of the Piltdown jaw and cranium, in which he reviewed Dr. Hrdlička's conclusions, which were largely based upon a study of the teeth, and gave a brief forecast of the results of the reconstruction at which he and Elliot Smith had arrived in their joint study of the cranium. The paper on the Neanderthoid Australian skull mentioned above is worthy of special attention, as it is not so well known as it deserves. The skull was that of a female and showed a remarkable combination of primitive character in the cranium, combined with more delicate characters in the facial region, which was both orthognathic and leptorrhine It had an enormous development of the supraciliary ridges, and glabella, a well-developed bregmatic eminence, and a marked development of the torus occipitalis. Added to these primitive features was an extremely low sloping forehead, while the maximum occipital point coincided with the inion. Certain features showed a resemblance to Pithecanthropus.

Vol. I of Series I2 (Social Science-Economics, Education, History, Philosophy, and Psychology) contains ten reprints, all short, varying from three to twenty-four pages, but there is no shame in brevity and these scholarly little articles are a valuable contribution to general philosophic and psychological knowledge. Among so many varied papers it is difficult to make a selection for special note. Some of the articles are more descriptive, others more constructive, and high in the first category comes Prof. E. E. Anderson's stimulating account of "The Present Religious Situation "- -a careful consideration of the nature of religious development. Sir John Macpherson's analysis of "The Influences which cause Fluctuations in the Production of Insanity" shows the enormous complexity of the subject, and the need for careful scrutiny of seemingly accurate statistics, and Prof. B. Muscio shows his characteristic originality and freedom from scholastic bias in his thoughtful contribution on "The Meaning of Philosophy." These reprints bear the same relation to the usual scientific treatise that a magazine of short stories bears to a novel, and, like a magazine, the volume is pleasantly readable.

Vol. I of Series I3 consists of twenty-three papers published in various journals during the years 1920 I924 by workers in the Department of Zoology of the University of Sydney. The series includes the late Prof. W. A. Haswell's last two papers-on Astacocroton - a new type of acarid parasitic on the gills of crayfishes, and on the structure of the proboscis of the syllid worms, and two papers by Prof. L. Harrison on the breeding habits and the pigmentation of the eggs of some Australian frogs. Mr. E. A. Briggs, lecturer in zoology, contributes two papers, one of which describes in detail a new species of crawling medusa, and Miss E. E. Chase, demonstrator, gives an account of a new avian trematode. The two Macleay fellows of the Linnean Society of New South Wales, Miss M. Henry and Miss $V$. Irwin-Smith, are responsible for the remaining papers. Four by Miss Henry are contributions to a monograph of the fresh-water Entomostraca of New South Wales, and those by Miss Irwin-Smith are accounts of nematode worms, especially from lizards, and of the life-history of brachycerous Diptera. The Department of Zoology in Sydney has from its institution played a worthy part in scientific research, and the volume affords good evidence that no time NO. 2936 , VOL. II 7 ] 
was lost after the conclusion of the War in resuming investigations.

Vol. I of Series 2 contains botanical papers published between I92I and I924. To the botanists in a new country falls the duty of widening our horizon as to botanical conditions, and these papers are valuable examples of such literature. They include ecological studies of Australian vegetation by Marjorie I. Collins, of mangrove and salt marsh vegetation and arid and semi-arid regions; studies of Australian parasitic flowering plants, and of Australian examples of symbiosis (mycorhiza and root-nodules) by J. M'Luckie, also notes upon the life-history and structure of Australian Phanerogams and ferns by Prof. Lawson, P. Brough, and Jessie Steele. M. M. Williams has a brief note upon interesting brown seaweeds, including the one apparent parasite in the group, Notheia anomala.

Series II covers mathematics, physics, and astronomy, and vol. I contains seven papers dealing mainly with physical questions. They show that the University is not restricting its research work to the industrial problems which arise in the colony, but is taking its part in the solution of scientific questions the interest of which is world wide. Mr. E.H. Booth deals with the ions produced in air which has bubbled through water, a process which bears on the problem of how the earth maintains its negative charge. Dr. G. Harker shows that the vapour arising from a boiling solution has a temperature higher than that from the pure solvent boiling under the same conditions. Prof. Carslaw solves a problem on the cooling of a sphere with a core and shows that in the Mathematical Tripos of I9O4 the candidates were asked to prove something which was untrue, while a paper by the late Prof Pollock gives an interesting account of the physics of the stethoscope.

Vol. I of Series 3 is a collection of papers published in the various chemical journals by workers in the chemistry departments of the University. These show that research work is being actively prosecuted. Physical chemistry measurements include those of the molecular solution volumes of various organic substances in ethyl alcohol, which show that several of the solutes are associated in the liquid state; the development of the miscibility tests for eucalyptus oils in which the oils are added to methyl alcoholwater mixtures and the critical solution temperatures determined; the decrease in the values of the reaction constant in the decomposition of hydrogen peroxide by colloidal platinum when different concentrations of protective colloids are added, and the action of soaps as protective colloids for gold hydrosols, in which it is shown that although the stabilisation is very pronounced the coagulating influence of the sodium ion of the lower soaps begins to outweigh the protective action. A number of the reprints deal with organic chemistry: the preparation of organic compounds of arsenic of the type $R_{1} R_{2} R_{3} A s$, $R A s I_{2}$ where $R_{1} R_{2}$, etc., indicate similar or dissimilar alkyl or aryl groups; studies in the formation of ring compounds by condensation of aromatic amines and diketones, etc.; researches on the phellandrenes and a number of contributions to the chemistry of piperitone. In addition, investigations have been carried out on the active principle of trees of the kind to which belong the dreaded " ordeal " or "doom tree" of the African pygmies.

\section{Voltaire and Medicine.}

$\mathrm{N}$ the second part ${ }^{1}$ of his paper on "Voltaire and Medicine," read before the Section of the History of Medicine of the Royal Society of Medicine on December 16 , the president, Dr. J. D. Rolleston, gave some account of Voltaire's allusions to anatomy and physiology, his advocacy of inoculation against small-pox, and his interest in the history and ravages of syphilis, a knowledge of which he had derived from Astruc's work on venereal diseases. He also referred to the attention paid by Voltaire to other matters connected with public health, his acquaintance with medical jurisprudence and particularly his sceptical attitude toward historical cases of poisoning, in many of which he showed that death was more probably due to some acute infection, and his remarks on various diseases of social importance, such as mental disorders, convulsive hysteria at the tombs of saints and ecclesiastics, alcoholism and the King's evil.

Several passages in his works show that Voltaire had paid some attention to anatomy. He commences his article on "Man" in the "Dictionnaire Philosophique" by saying that in order to learn the physical aspects of the human race, one must study works of anatomy or the article of $\mathrm{M}$. Venel in the "Dictionnaire Encyclopédique," or rather follow a course of anatomy. In his article on "Anatomy" in the same work, he remarks that "Ancient anatomy bears to modern anatomy the same relation that the rough geographical charts of the sixteenth century do to the topographical maps of to-day." In the "Histoire du Docteur Akakia" Maupertuis is condemned for his advice to neglect anatomy. Voltaire, however, fully realised that much was yet learned

${ }^{1}$ See NATURE, December I9, I925, p. 9 I9.

NO. 2936 , vOL. II 7 ] in human anatomy. In Chap. 35, entitled " Incertitudes en Anatomie" of the essay "Des singularités de la nature," he exclaims, "In spite of all the help that the microscope has given anatomy, and in spite of the great discoveries of so many skilful surgeons and physicians, what interminable discussions have arisen, and in what uncertainty we still remain!",

Although physiology was still in its infancy in the eighteenth century, Voltaire seems to have been well abreast of the knowledge of his time. In the "Dictionnaire Philosophique" he alludes to the uncertainties current in connexion with circulation, digestion, generation, and muscular tonicity, and refers to the pioneer work of Borelli, Keil and Jurin in cardiac physiology. He exposes the errors of Descartes regarding digestion and circulation, and heaps ridicule on the casuist Sanchez and the philosopher Maupertuis for their extraordinary views on generation and embryology.

Voltaire's interest in public health was shown not only by his desire to control small-pox by inoculation, and syphilis by a league of nations to combat the disease - a project not realised until the formation of the Union internationale contre le péril vénérien in I923-but also by his allusion to other epidemic diseases, especially plague and malaria, and his condemnation of the insanitary condition of the Paris hospitals, in which overcrowding and cross infection were rife. He also showed his concern for public health in his references to the abuses connected with the administration of military hospitals, the congested state of the Paris cemeteries and the practice of burial in churches, as well as by his proposal to found maternity hospitals for unmarried women. 